\title{
New insights into diffusion in 3D crowded media by Monte Carlo simulations: effect of size, mobility and spatial distribution of obstacles
}

\author{
Eudald Vilaseca, ${ }^{1 *}$ Adriana Isvoran, ${ }^{2}$ Sergio Madurga, ${ }^{1}$ Isabel Pastor, ${ }^{1}$ \\ Josep Lluís Garcés ${ }^{3}$ and Francesc Mas ${ }^{1}$
}

\author{
${ }^{1}$ Physical Chemistry Department and Research Institute of Theoretical and \\ Computational Chemistry (IQTCUB) of Barcelona University (UB), \\ C/ Martí i Franquès, 1, 08028-Barcelona, Catalonia, Spain \\ ${ }^{2}$ Department of Chemistry, University of the West Timisoara, Timisoara, Romania, \\ ${ }^{3}$ Chemistry Department, Lleida University (UdL), Lleida, Catalonia, Spain
}

\begin{abstract}
Particle diffusion in crowded media was studied through Monte Carlo simulations in 3D obstructed lattices. Three particular aspects affecting the diffusion, not extensively treated in three-dimensional geometry, were analysed: the relative particle-obstacle size, the relative particle-obstacle mobility and the way of having the obstacles distributed in the simulation space (randomly or uniformly). The results are interpreted in terms of the parameters that characterize the time dependence of the diffusion coefficient: the anomalous diffusion exponent $(\alpha)$, the crossover time from anomalous to normal diffusion regimes $(\tau)$ and the long time diffusion coefficient $\left(D^{*}\right)$. Simulation results indicate that there is a more anomalous diffusion (smaller $\alpha$ ) and lower long time diffusion coefficient $\left(D^{*}\right)$ when obstacle concentration increases, and that, for a given total excluded volume and immobile obstacles, the anomalous diffusion effect is less important for bigger size obstacles. However, for the case of mobile obstacles, this size effect is inverted yielding values that are in qualitatively good agreement with in vitro experiments of protein diffusion in crowded media. These results underline that the pattern of the spatial partitioning of the obstacleexcluded volume is a factor to be considered together with the value of the excluded volume itself.
\end{abstract}

Key words: anomalous diffusion; macromolecular crowding; obstructed diffusion; Monte Carlo simulation.

\footnotetext{
* Corresponding author

e-mail: eudald.vilaseca@ub.edu
} 


\section{Introduction}

The interior of the living cell is highly concentrated and structured with molecules having different shapes and sizes, up to $40 \%$ of its total mass being represented by macromolecules $^{1-3}$. The high concentration of macromolecules in intracellular environments results into non-specific interactions (macromolecular crowding), which have a great influence on the kinetics and thermodynamics of possible reactions that occur in these systems, e.g. diffusion processes and reaction kinetics ${ }^{3-15}$. Diffusion is a basic transport mechanism that is presented in a wide range of complex systems including living cells and it has strong connections with a lot of phenomena of crucial importance for sustaining life. However, macromolecular crowding has been shown to alter molecular diffusion both quantitatively and qualitatively $y^{3,5,12-14}$. Quantitatively, macromolecular crowding reduces the diffusion coefficient as compared to aqueous solutions and, qualitatively, diffusional motion can change to anomalous diffusion, what means a time dependent diffusion.

A great deal of information about motion of molecules in living cells has been obtained from intracellular measurements using different experimental techniques ${ }^{13,16-}$ 35 and from simulations ${ }^{14,36-53}$. Experimental data are usually obtained by fluorescence recovery after photobleaching (FRAP) and fluorescence correlation spectroscopy (FCS) techniques. In order to give only a few examples, FRAP experiments have revealed anomalous diffusion of dextrans ${ }^{17-18,23}$, of proteins in cytoplasm ${ }^{19,21-22,25,33}$ and of proteins in dextrans ${ }^{35}$. FCS experiments have also shown anomalous diffusion of nanoparticles with different sizes in agarose gel ${ }^{26}$, of dextrans in HeLa cell cytoplasm $^{28}$, of proteins in three-dimensional crowded media ${ }^{27,31}$, of Alexa488 lightemitting particles in extracellular matrices ${ }^{32}$, respective of the inert gold nanoparticles in the cytoplasm and nucleoplasm under various stress conditions ${ }^{34}$. 
However, there is not still a complete explanation of transport processes in living systems and simulation methods can be useful to help understanding them. Most of the published simulation studies concerning diffusion processes in biological systems are developed for two-dimensional media (2D). These simulations, and in particular the extensive work of Saxton ${ }^{14,36-37,39-41,51}$ have shown that, in crowded media, diffusion presents two behaviours: it is anomalous (time dependent diffusion coefficient) for short times and normal (constant diffusion coefficient) for long-times. With respect to the diffusion coefficient value, it has been shown that: it diminishes when the concentration of obstacles increases ${ }^{41,51}$, it is dependent on the relative mobility of tracers and obstacles ${ }^{39-41,52}$, and it also depends on the size of tracers and obstacles $^{36-37,50}$. In addition, the results show that in a uniform distribution of the obstacles the diffusion coefficient is greater than in a random distribution ${ }^{50}$. However, for a correct interpretation of these results, it has to be considered that in $2 \mathrm{D}$ crowded media the anomalous diffusion is due to two factors: the low dimensionality of the system and the molecular crowding. Thus, the assessment of the real importance of the crowding in the diffusion phenomena requires a systematic analysis of diffusion in three-dimensional media $(3 \mathrm{D})$, where the topological space restrictions are not present.

Although the literature concerning 3D simulation studies on diffusion processes in biological systems is less extensive, there are several works that must be emphasized. For example, Netz and Dorfmuller ${ }^{42}$ performed a Monte Carlo study of the diffusion of hard spheres in a restricted geometry, which give us insights into the factors that lead to anomalous diffusion, showing that the influence of tracer size is complementary to the influence of obstacle concentration on the diffusion processes. In another Monte Carlo simulation in $3 \mathrm{D}$ crowded media, Olveczki and Verkman ${ }^{43}$ 
have shown that anomalous diffusion of solutes in organelles is strongly dependent on organelle structure. Lipkow and his coworkers ${ }^{46}$ have developed a computational program, Smoldyn, for studying cellular molecular processes taking into account both the spatial location of proteins (and their complexes) and their diffusive motion. This program has shown results in good agreement with experimental data for diffusion of signaling protein CheYp through the cell. The analysis by Brownian simulation of single-molecule trajectories followed by a Monte Carlo simulation of nonreactive fluorophore, developed by Dix et al. $^{47}$, revealed anomalous subdiffusion for fluorescently labeled molecules in 3D complex media. Echeveria and his coworkers ${ }^{48}$ have used a mesoscopic algorithm to simulate the diffusion and reactions of small particles in a 3D medium with obstacles. They analyse how the diffusion coefficient value diminishes with the total excluded volume. More recently, Saxton has shown that diffusion is anomalous for all times if particles diffuse in a suitable hierarchy of binding sites and are not in thermal equilibrium with traps ${ }^{14}$.

Apart from the considerations of these simulation results, there are other factors that must also be considered. Dix and Verkman ${ }^{13}$ after an exhaustive analysis of literature, conclude that the role of molecular crowding is not so important to determine the anomalous diffusion and that the slowing of diffusion in cells is really less marked than it is generally assumed. Another point of view is expressed by the review of Zhou, Rivas and Minton ${ }^{12}$. They affirm that, within living cells fast associations are under diffusion control and slow associations are under reaction control. Also, macromolecular crowding usually decelerates fast associations and accelerates slow ones. From their point of view, biological fluids are more complex than systems usually considered in simulation due to the nonspecific interactions and it is necessary to obtain well-defined model systems for further theoretical investigations. Moreover, from several experimental works, it is well known that the 
anomalous diffusion emerges on cytoplasmatic macromolecules and it depends on the size and conformation of the tracer particle and on the total protein concentration of the solution ${ }^{28}$. There are even experimental studies that show anomalous protein diffusion in in vitro, with the anomalous diffusion exponent decreasing continuously with increasing obstacle concentration and molecular weight ${ }^{31,35}$.

The aim of the present study is to perform a series of Monte Carlo simulations of small particle diffusion in 3D obstructed lattices in order to assess the real effect of macromolecular crowding on the diffusion without the interference of the low dimensionality restrictions that appear in two-dimensional systems. We have focused on three particular aspects that were not extensively treated in previous studies. We have quantified the effect of the obstacle size, their relative mobility and the manner the obstacles are distributed (at random or uniformly). Although the role of these factors has been previously analysed in $2 \mathrm{D}$ media $^{36-37,39,40,52}$, a systematic threedimensional study that quantifies their importance without the influence of the low dimensionality restrictions was lacking. Moreover, a new contribution of this work is the study of how diffusion is affected by the different ways of partitioning a same obstacle-excluded volume: from a great quantity of small size particles to a small quantity of great size particles. In fact, the pattern of the spatial distribution of the volume excluded by obstacles is an additional aspect to be considered together with the value of the excluded volume itself.

The paper is organized as follows: methods, results and discussion, and conclusions. "Methods" section contains three subsections: a theoretical background on the mathematical concepts of anomalous diffusion, a subsection presenting the simulation algorithm and another subsection presenting how we obtain the values of the investigated parameters for the quantitative analysis. The "Results and discussion" section is also divided into three subsections emphasizing the effects of 
obstacle concentration and sizes, of the manner they are distributed and of their mobility. The main outcomes of the study are summarized in the "Conclusions" section.

\section{Methods}

\section{A. Theoretical background}

A diffusion process taken by a solute in dilute solutions can be described with the well-known Einstein-Smoluchowski equation:

where $d$ is the topological dimension of the medium where the process is embedded and $D$ is the solute diffusion coefficient ${ }^{54-56}$. In crowded media, typically in in vivo and in a great number of in vitro processes, the existence of different macromolecular species, proteins, nucleic acids, organelles, etc., hinders the diffusion process. In these cases, eqn (1) must be generalized to deal with a more complex process, known as anomalous diffusion ${ }^{16,31,55-56}$ which can be described by:

where $\alpha$ is defined as the anomalous diffusion exponent $(0<\alpha<1$ is the case of subdiffusion and $\alpha>1$ holds for the case of superdiffusion) and $\Gamma$ is a generalized transport coefficient, also known as anomalous diffusion coefficient, of units $\left[\right.$ length $^{2} /$ time $\left.^{\alpha}\right]$ which value depends on the degree of crowding in the medium. This definition allows us to introduce a generalized time-dependent diffusion coefficient function, as: 
where represents the excluded volume given by the different macromolecular species present in the solution and determines its degree of crowding. Then, eqn (2) can be written as:

In order to work with dimensionless magnitudes it is usual to introduce some characteristic length unit, , which is related to the mean free path of the solute and it can be associated to the unit length of the simulation lattice, and some characteristic time unit, which is the jump time and it can be associated to the unit time of the simulation process. Therefore, eqn (4) becomes:

where, and are the dimensionless length and the dimensionless time, respectively, $\quad$, is the dimensionless time-dependent diffusion coefficient function, and is the diffusion coefficient of the solute in solution without crowding. This value is related to the units of length and time by the EisnteinSmoluchowski eqn (1) as

From, now, in order to simplify the notation, we drop the tilde in , and Then, eqn (5) is now written as

which is the dimensionless form of eqn (4). It must be taken into account that the $2 d$ factor only appears in the dimensioned form of the generalized EinsteinSmoluchowski diffusion equation. 
Experimental and theoretical data ${ }^{14,23,25,27-28,31,39-41}$ reveal that, in crowded media, there is a succession of diffusion behaviours that can be identified with the three distinct regions observed in the $\log \left(<r^{2}>/ t\right)$ versus $\log (t)$ plots:

i) For really short times $\log \left(<r^{2}>/ t\right)$ is almost time independent reflecting that the diffusion process is not yet affected by macromolecular crowding. We define this initial value as

and the dimensionless value of is in accordance with the value for the case without crowding.

ii) Anomalous diffusion corresponds to an intermediate region where, according to eqn (3), $\log \left(<r^{2}>/ t\right)$ linearly decreases with a slope $\alpha-1$.

iii) For long-times, $\log \left(<r^{2}>/ t\right)$ tends to be constant again, reflecting a normal diffusion in a homogeneous dense medium with a diffusion coefficient $\left(D^{*}\right)$ lower than that corresponding to a dilute solution

and the dimensionless value of is in accordance with the value for the case without crowding. The shifting from the anomalous diffusion regime to the normal one is characterized by the crossover time, $\tau$. 
The characteristic parameters of the $\log \left(<r^{2}>/ t\right)$ versus $\log (t)$ curves: anomalous diffusion exponent, $\alpha$, limiting diffusion coefficient, $D^{*}$, and crossover time, $\tau$, have been considered in our quantitative analysis of diffusion.

\section{B. Simulation algorithm}

Diffusion in 3D obstructed media was modeled as a random walk process in which the randomly distributed diffusing particles, called tracers $(T)$, move in a 100x100x100 cubic lattice with cyclic boundary conditions and containing randomly distributed obstacles $(\mathrm{O})$ such as their density is under the percolation threshold. It has been checked that the simulation results are not dependent on the lattice size. Several test simulations performed for 50x50x50, 100x100x100 and 200x200x200 systems yielded the same results (simulations not shown here).

We consider only excluded volume interactions (hard-sphere repulsions), so any site in the lattice may not be occupied by two particles at the same time. Our approach is based on the experimental results obtained by Kao and coworkers ${ }^{38}$ and Wachsmuth and coworkers ${ }^{25}$, which have shown that probe collisions with intracellular components were the principal diffusive barriers that slowed the translational diffusion of small solutes.

Each tracer occupies a single site in the lattice. In contrast, to account for the usual greater size of the crowding molecules and to analyse the effect of the paticleobstacle relative size in the diffusion, four different sizes have been considered for obstacles (see Fig. 1): 1 site, 27 sites (a $3 \times 3 \times 3$ site cube), 81 sites (a cube of $5 \times 5 \times 5$ sites where the sites at the edges and near the vertex have been removed to obtain a quasi spherical shape) and 179 sites (a 7x7x7 site cube with the edge and vertex sites removed). We use the notation $5 \times 5 \times 5 \mathrm{R}$ and $7 \times 7 \times 7 \mathrm{R}$ (where $\mathrm{R}$ stands for rounded) to refer to the last two sizes. As every obstacle occupies many sites within the lattice, in our calculations we distinguish the density of sites occupied by obstacles, $[\mathrm{O}]_{\text {sites }}$ from 
the concentration of obstacle particles, [O]. We will refer to the density of sites occupied by obstacles, $[\mathrm{O}]_{\text {sites, }}$, as the excluded volume due to the obstacle presence $(\phi)$. Four values for the obstacle excluded volume have been considered: $0.1,0.2,0.3$ and 0.4. We must underline that because of the mutual spatial exclusion among tracers, the total excluded volume of the system is the sum of $\phi$ plus the volume occupied by the tracers. However, as the tracer excluded volume is the same in all the performed simulations (0.01) and smaller than the obstacle one, all the study will we referred to the obstacle excluded volume, $\phi$.

In the present study, the following situations have been considered:

i) tracers $([\mathrm{T}]=0.01)$ in lattices with randomly distributed immobile obstacles with different site density occupancy: $\phi=0,0.1,0.2,0.3$ and 0.4 . We have chosen these densities in agreement with experimental data concerning to the range of macromolecular crowding agents in cytoplasm,

${ }^{1,2}$. These simulations were carried out for the four obstacle sizes shown in Fig. 1.

ii) tracers $([\mathrm{T}]=0.01)$ in lattices with mobile obstacles. The mobility is controlled by a probability factor $M$ that determines whether an obstacle is allowed to move after being randomly selected. The following $M$ values were considered: $0.25,0.5,0.75$ and 1 . This series of simulations were done for the obstacle size and obstacle excluded volume values considered in the i) calculations.

iii) tracers $([\mathrm{T}]=0.01)$ in lattices with uniformly distributed immobile obstacles. In this case the obstacles form a cubic regular lattice with a constant separation between every pair of nearest-neighbours. The obtained results are compared with those given by the simulations with a random distribution of obstacles. To make the comparison reliable the space between two nearest-neighbour obstacles in the uniform distribution and the size of the simulation lattice is 
defined for each case so that the obstacle site density is the same for both, uniform and random, obstacle distribution models. The considered uniform distributions are presented in Table 1.

At every time step a random number is used to choose a particle to move (a tracer or an obstacle, if mobile). For a tracer, the destination site is randomly chosen among the 6 nearest-neighbours of the origin site. If the proposed site is empty the considered particle moves to it, otherwise it remains in its initial position and another particle is randomly chosen to move. For a big size obstacle, the central site is proposed to move one position in one of the six spatial directions, randomly chosen. The obstacle displacement is done if the new sites to be occupied are empty. For each Monte Carlo time step this sequence is repeated $\mathrm{N}_{\text {tot }}$ times $\left(\mathrm{N}_{\text {tot }}\right.$ is the total number of mobile particles within the lattice) in order to assure that statistically each molecule moves once in the time step. Every simulation run lasted 10000 time steps and every run was repeated from 100 to 400 times with a different initial particle disposition. For each case, the mean squared displacements are averaged along these repetitions. This algorithm was implemented in a fortran program.

\section{C. Characteristic parameters of the diffusion process}

The time dependence of the diffusion coefficient is analysed, according to eqn (3), with the $\log \left(<r^{2}>/ t\right)$ versus $\log (t)$ curves obtained from the computer simulations. From each curve the three characteristic parameters of the diffusion are extracted: the anomalous diffusion exponent $(\alpha)$, the crossover time $(\tau)$ and the long-time diffusion coefficient $\left(D^{*}\right)$. In Fig. 2 it is shown how these parameters are obtained.

We notice in Fig. 2 that for an unobstructed lattice $(f=0$, homogenous media) the plot is a horizontal line, indicating that diffusion coefficient is constant. The 
$\log \left(<r^{2}>/ t\right)$ value is not exactly zero because there is a small autocrowding effect due to the hard sphere tracer repulsions. In contrast, the curves corresponding to the obstructed lattices $(f=0.3$ and 0.4$)$ present two characteristic regions. There is a region with a linear decreasing of $\log \left(<r^{2}>/ t\right)$, which corresponds to an anomalous diffusion behaviour, followed by a region with a smaller constant diffusion coefficient $\left(D^{*}\right)$ characteristic of normal diffusion. It should be noticed that, as it was reported in other studies $^{17,36-41}$, the initial region of normal diffusion (when the diffusing particles are not still affected by the crowding obstacles) is not observed in the present onlattice simulations. The plot starts at a position, $D_{0}{ }^{\prime}(\phi)$, lower than $D_{0}(\phi)$ (the initial normal diffusion coefficient given by eqn (7) not observed in the simulation), which value depends on the discretization of the lattice, the obstacle size and the excluded volume, and immediately decreases to reach the linear anomalous diffusion region.

According to eqn (3), the value of the anomalous diffusion exponent is calculated from the slope of the linear time decreasing region of the $\log \left(<r^{2}>/ t\right)$ versus $\log (t)$ plot. The long-time diffusion coefficient is the long-time asymptotic limit of the plot. Finally, as it is illustrated in Fig. 2, the crossover time is given by the intersection of the linear fitting of the anomalous diffusion region and the horizontal line corresponding to the limiting diffusion coefficient of the normal diffusion region $^{41}$.

The crossover length, defined as , is another characteristic parameter of the diffusion process that has been analysed. Its value represents the minimum displacement distance from which the diffusion becomes again normal. It is related with the characteristic length of the obstacle distribution. Tracer displacements greater than this characteristic length see the obstacle distribution structure as a homogeneous medium. 
The $\left\langle r^{2}>\right.$ versus $t$ plots have also been analysed. According to eqn (6), for the normal diffusion region this plot is a straight line with a slope proportional to its corresponding normal diffusion coefficient. As, in the simulations, the crossover time from the anomalous to the normal diffusion regions occurs very early, most of the plot corresponds to the straight line of this final normal diffusion region (Fig. 3). The initial normal diffusion and the anomalous diffusion regions occupy a very short interval. This is the reason why a linear fitting in these plots usually yields the value of the limiting diffusion coefficient $\left(D^{*}\right)$. We have used these plots to confirm the value of the limiting diffusion coefficient $\left(D^{*}\right)$ obtained from Fig. 2. As it will be discussed below, Fig. 3 also shows that for a fixed obstacle excluded volume the slope of the $<r^{2}>$ versus $t$ plot changes with the size of the obstacles.

\section{Results and discussion}

\section{i) Effect of obstacle density and size}

In a first series of simulations, two different aspects of the crowding effect on diffusion have been analysed: the effect of the obstacle excluded volume (density of sites occupied by obstacles) and, for a fixed value of the obstacle excluded volume, the effect of the spatial distribution of the occupied sites (different obstacle sizes). This second aspect gives a new insight into the excluded volume analysis, since, usually, studies on crowding effects on diffusion consider variations of particleobstacle relative size without keeping the total excluded volume at a fixed value.

Fig. 4a shows the dependence of the anomalous diffusion exponent on obstacle excluded volume for obstacles having different sizes. There it can be seen that for a given obstacle size the anomalous diffusion exponent decreases when the obstacle excluded volume (the number of obstacle particles in this case) increases. That is, for a greater excluded volume there is a stronger obstruction of the diffusion 
process causing a marked decreasing of the diffusion coefficient with the time. This conclusion is qualitatively similar to that of Monte Carlo simulations of tracer diffusion in $2 \mathrm{D}$ crowded media ${ }^{41,49-50,52}$. It should be noted that the anomalous diffusion exponent values obtained in the present simulations fall within the 0.9-1 interval, whereas, in $2 \mathrm{D}$, it attains smaller values ( 0.7 in ref. 40$)$ because the low dimensionality restrictions to diffusion are added to the obstruction caused by the crowding. Higher values were obtained in experimental studies on 3D diffusion in agarose gels ${ }^{26}$. On the other hand, FCS experiments on streptavidin protein diffusion in dextrans solutions ${ }^{31}$ and FRAP measurements of alpha-chymotrypsin diffusion also in dextrans ${ }^{35}$ yielded lower anomalous diffusion exponent values (until 0.75). We think that in order to reproduce these low $\alpha$ values, the Monte Carlo simulation model needs to consider additional aspects of the real system such as hydrodynamic and/or intermolecular interactions.

An important result that can be observed in Fig. 4a is that for a given value of the obstacle excluded volume $(\phi)$ the smallest anomalous diffusion exponent corresponds to the case where the obstacles have the smallest size. This means that a given total excluded volume, due to obstacles, produces a higher crowding when it is divided into a greater number of smaller pieces (small size obstacles). In contrast, smaller crowding effect is observed when the same excluded volume is divided into a smaller quantity of bigger pieces (big size obstacles). In that case the tracers find greater empty spaces to move without colliding with an obstacle and there are fewer barriers to diffusion. These results confirm that not only the value of the total excluded volume but also its spatial distribution pattern in the simulation lattice determines the diffusion process.

Fig. $4 \mathrm{~b}$ shows the diffusion coefficient values for the long-time normal diffusion obtained from the computer simulations. $D^{*}$, defined by eqn (8), is 
expressed in terms of the $<r^{2}>/ t$ relation, according to the relative diffusion coefficient $D_{\phi}$ given by eqns (3)-(6). Our calculations give, in fact, a $D^{*}$ value of 0.995 for $\phi=0$ because of the autocrowding due to the tracer hard sphere repulsions. In Fig. $4 \mathrm{~b}$ it can be seen that for a given obstacle size the smaller $D^{*}$ values are attained at higher obstacle densities. The plot shows that, for the considered excluded volume interval, this variation is almost linear. These results confirm that the increase of the excluded volume reduces the tracer diffusion. On the other hand, if one considers a given obstacle excluded volume $(\phi)$, the long-time diffusion coefficient value is smaller when the excluded volume due to obstacles is divided into a greater number of smaller obstacle particles. This fact is in accordance with the more obstructive effect of a more partitioned crowding. The comparison of Fig. $4 \mathrm{a}$ and $4 \mathrm{~b}$ shows that the values of long-time diffusion coefficient, $D^{*}$, are correlated with those of the anomalous diffusion exponent, $\alpha$. The greater the slope of the anomalous diffusion region is, a smaller long-time diffusion coefficient is attained.

In Fig. $4 \mathrm{c}$ the crossover time, $\tau$, values obtained for these situations are also collected. There it can be seen that for a given obstacle excluded volume $(\phi)$, the crossover time from anomalous to normal diffusion occurs later for big obstacle particles and earlier for small ones. In contrast to small size obstacles, for big ones there are a smaller number of obstacle particles and thus a greater mean distance between them. As a consequence, the diffusing particles need more time steps to perform displacements greater than the characteristic length of the obstacle distribution. For small size obstacles the time needed to "see" the obstacle distribution as a homogeneous system is shorter. The crossover time differences between small and big size obstacles are progressively reduced as the obstacle excluded volume increases. 
Fig. 4c also shows, for each obstacle size, the dependence of the crossover time value on the obstacle excluded volume. It can be seen that there are different behaviours. For the small size obstacles the crossover time increases with the obstacle concentration, whereas for the biggest ones the crossover time diminishes. For a single site obstacle, when increasing the number of particles, the mean distance among obstacles diminishes and the characteristic length of the obstacle distribution can be estimated to be shorter. Thus, it could be expected that the normal diffusion regime is reached at shorter time intervals. However, as simultaneously the obstruction to diffusion is greater, there is a delay in the time when the diffusing particles see the obstacles as a homogeneous distribution. The final position of the crossover time is a consequence of the competition of these two factors. In systems with small obstacles the reduction of the diffusion prevails over the reduction of the mean distance among obstacles. Our results are similar to those given by Saxton for point obstacles in $2 \mathrm{D}$ diffusion ${ }^{40}$. On the other hand, for big size obstacles the balance is the reverse, as the increase in the obstacle density needs a smaller increment of obstacle particles the obstruction to diffusion is less relevant than the reduction of the mean distance among obstacles.

The calculation of the crossover length, , shown in Fig. 4d, confirms this different behaviour. For each obstacle size there is an almost linear dependence of the crossover length on the obstacle size and excluded volume. As mentioned before, this parameter can be interpreted as the minimum displacement length for the tracer to see the obstacle distribution as a homogeneous medium. The density of occupied sites around an average empty site of the simulation lattice (Fig. $5)$ indicates that the $r^{*}$ values are situated in the region where this function becomes constant. 
From these results we should notice the distinct behaviour of the 1-site obstacles. For the anomalous diffusion exponent and the long-time diffusion coefficient these obstacles give a markedly smaller values that can be interpreted in terms of its relative smaller size. On the other hand, 1-site obstacles are the only ones that increase the crossover time and length parameter values with the excluded volume. This behaviour, which was also observed in the Saxton simulations of 2D diffusion $^{40}$, is related with the greater proportion of obstacle clusters that present the smaller size obstacles.

As a complementary study, the values of the initial $(t \rightarrow 0)$ diffusion coefficient, $D_{0}{ }^{\prime}(\phi)$, obtained in the different simulations were analysed (Table 2). The results indicate that the $D_{0}{ }^{\prime}(\phi)$ value depends on the obstacle size and excluded volume and is similar to the value given by 1 minus the probability that a tracer, in a single jump, reaches an occupied site. As this probability is computed assuming that all obstacles are separated among them, it value gives an upper limit estimation for the initial $D_{0}{ }^{\prime}(\phi)$.

Although these simulation results are coherent and in agreement with $2 \mathrm{D}$ simulations $^{36-41,44-47}$, there are experimental $3 \mathrm{D}$ studies $^{31,35}$ that show a reverse result for the limiting diffusion coefficient and the anomalous diffusion exponent values. Whereas our simulations, performed with randomly distributed immobile obstacles, indicate that, for a given excluded volume, the small size obstacles are more obstructive to diffusion and yield a smaller value for $D^{*}$ than the big size ones, the experiments, performed in in vitro with mobile obstacles, show an opposite ordination of $\alpha$ and $D^{*}$ values indicating that, in reality, the small size obstacles are the less obstructive to diffusion. This different behaviour can be attributed to the obstacle mobility that has not been considered in these simulations. In fact, in a real solution, the crowding molecules can move and, consequently this fact may change their 
crowding effect on diffusion. In the next section we explore how these results may change when obstacles are allowed to move.

\section{ii) Effect of obstacle mobility}

In the simulation model the degree of mobility of an obstacle is defined by the probability factor $\mathrm{M}$, which determines whether an obstacle is tried to move after being randomly selected. The values $0,0.25,0.5,0.75$ and 1 have been considered. In fact, $M$ represents the relative jump probability of obstacles with respect to tracers and gives an indication of their approximate relative diffusion coefficient. It should be noted that $\mathrm{M}$ is not the exact relative diffusion coefficient because this parameter can only be determined from the particle displacements observed during the simulation.

The effect of obstacle mobility on tracer diffusion can be observed in Fig. 6 where the $\log \left(<r^{2}>/ t\right)$ versus $\log (t)$ curves for the case of obstacles having a size of 81 sites $(5 \times 5 \times 5 R)$ have been plotted. These curves show that increasing the obstacle mobility reduces the obstruction effect to diffusion. For a greater mobility $(M=1)$ the reduction of the diffusion coefficient with time is slower (greater $\alpha$ ), the anomalous diffusion region is shortened (smaller $\tau$ ) and the diffusion coefficient, $D^{*}$, for the long-time normal diffusion is greater. The more obstructive effect to diffusion is obtained for fixed obstacles. Similar plots are obtained for the other obstacle sizes.

As in experimental systems the mobility of crowding molecules is inversely proportional to their size, we have analysed the obstacle obstructive effect to diffusion by assigning a different mobility factor $\mathrm{M}$ to each obstacle size. To obtain a qualitative indication of the different size-mobility effect, a value of $\mathrm{M}=0$ have been given to the 179 -site obstacles, $M=0.25$ for the 81 -site ones, $M=0.75$ for obstacles having 27 sites, and the maximum mobility $(M=1)$ for the single site obstacles. The 
diffusion characteristic parameters obtained from the simulation curves are shown in Fig. 7. As the mobility reduces the obstructive effect of obstacles, the differences among the diffusion parameter values are smaller. However, in comparison to what happens with immobile obstacles, some significant changes are observed.

The obstacle mobility increases the value of the anomalous diffusion exponent, $\alpha$, that is, the reduction rate of the diffusion coefficient with time is smaller. Moreover, as a result of the different obstacle mobilities we observe that, for each obstacle excluded volume, the anomalous diffusion exponent is greater for the small size obstacles and smaller for the big ones (Fig. 7a). Due to their greater mobility, the smaller obstacles produce a lesser obstruction to tracer diffusion. This new ordering of $\alpha$ values with respect to obstacle size is the reverse of what is observed in immobile obstacles (Fig. 4a) and coincides with experimental measurements ${ }^{31,35}$. The qualitative agreement with these experiments indicates that obstacle mobility must be taken into account in the interpretation of diffusion processes.

The limiting diffusion coefficient, $D^{*}$ (Fig. 7b), shows a similar behaviour. First, the obstacle mobility increases the $D^{*}$ values as a consequence of a smaller obstruction to tracer diffusion. Second, due to the different obstacle mobilities, the ordering of $D^{*}$ values with respect to obstacle size is also the reverse of the ordering for immobile obstacles (Fig. 4b). Now, despite that the $D^{*}$ value differences are small, for a given obstacle excluded volume, the larger obstacles have a smaller diffusion coefficient. Only the case of single site obstacles shows a particular behaviour. In spite of its mobility $(\mathrm{M}=1)$, the greater obstructive effect of this highly partitioned excluded volume yields diffusion coefficient values smaller than the other obstacles.

Another effect of the obstacle mobility is the reduction of the crossover time (Fig. 7c), that is, the final regime of normal diffusion is reached earlier. The 
comparison of Fig. 7c with Fig. 4c (immobile obstacles), shows that the obstacles with a greater mobility: $1 \times 1 \times 1(M=1)$ and $3 \times 3 \times 3 \quad(M=0.75)$, present a marked reduction of the crossover time and show a value that is constant for all the obstacle excluded volumes. It can be interpreted that the obstacle mobility tends to homogenize the system reducing the transition time to the final normal diffusion. For the obstacles with smaller mobility $(\mathrm{M}=0.25)$ the change is in the same direction but the reduction of $\tau$ is less acute (Fig. 7c).

A similar situation is observed for the crossover length parameter (Fig. 7d). The obstacles with a greater mobility have smaller crossover length values than when they are immobile, being these values almost independent of the total obstacle excluded volume. As the crossover length is related to the characteristic length of the obstacle distribution, this results means that in the case of mobile obstacles this characteristic length is independent of the obstacle excluded volume and it depends only on the obstacle size.

\section{iii) Random versus uniform distribution of obstacles}

Cellular environment also presents regions with high spatial organization, such as microtubules. In order to model small particle diffusion within these regions we have performed simulations with uniformly distributed obstacles forming a cubic lattice with a small separation (1, 2 or 3 sites) between nearest-neighbours (Table 1).

Fig. 8 shows the $\log \left(<r^{2}>/ t\right)$ versus $\log (t)$ curves obtained in these conditions for the 81-site obstacles $(5 \times 5 \times 5 \mathrm{R})$. The curves are compared with those obtained from diffusion in lattices with immobile randomly distributed obstacles. In both models two obstacle excluded volumes have been considered: $\phi=0.236$ and $\phi=0.375$.

We notice that, for a given obstacle density, the time decreasing of the diffusion coefficient is more accentuated when the immobile obstacles are randomly 
distributed. Similarly, for the random obstacle distribution the anomalous diffusion region is longer than for the uniform distribution and the crossover from anomalous diffusion to normal one takes place at a later time. In addition, the constant diffusion coefficient for long times is, for the uniform distribution of obstacles, always higher than for the random distribution. These results indicate that, for a fixed obstacle excluded volume, the uniform distribution of obstacles is lesser obstructive to small particle diffusion than their random distribution. The channels between obstacles of the uniform distribution give to the diffusing particles a continuum empty space where to move without obstructions. On the other hand, the random distribution produces a greater partitioning of the free space obstructing the communication between empty local volumes. A similar behaviour was observed in previous calculations of single particle diffusion in $2 \mathrm{D}$ crowded media ${ }^{49-50}$.

The effect of the spatial (random versus uniform) distribution of immobile obstacles on the tracer diffusion is analysed in Fig. 9 in terms of the 4 characteristic parameters of diffusion. For each obstacle size, 2 or 3 obstacle excluded volumes have been considered (Table 1). For the uniform distribution the highest $\phi$ value corresponds to a distribution in which the nearest-neighbour obstacles are separated by 1 empty site and the second $\phi$ value corresponds to a separation of 2 empty sites. The third $\phi$ value considered in the case of $3 \times 3 \times 3$ sites obstacles corresponds to a separation of 3 empty sites between nearest-neighbour obstacles. To make the comparisons more reliable, the simulations with randomly distributed obstacles have been done at the same excluded volume values as in the uniform distributions.

Fig. 9a and $9 \mathrm{~b}$ confirm that in all cases the uniform distribution is less obstructive to diffusion. The smaller size obstacles $(1 \times 1 \times 1$ and $3 \times 3 \times 3)$ show a greater change in the $\alpha$ and $D^{*}$ parameter when the distribution changes from random to uniform, and this change becomes more important at higher excluded volumes. The 
parameter value differences are smaller for the bigger obstacles $(5 \times 5 \times 5 \mathrm{R}$ and $7 \times 7 \times 7$ R). It should be noted that the results for uniformly distributed obstacles approaches the results found for mobile obstacles (Fig. 6) because the obstacle mobility tends to homogenize the obstacle distribution.

A particular result for the $\alpha$ values is observed in the uniform distributions. For the smaller obstacles the value of $\alpha$ increases with the excluded volume, that is, when the separation between two nearest-neighbours changes from 2 to 1 . Whereas for the bigger obstacles $\alpha$ decreases. This result could be related with the fact that smaller size obstacles that have a cubic shape and the bigger ones have a quasispherical shape.

In Fig. 9c and 9d, it can be seen that the uniform distribution of obstacles yields smaller crossover time and crossover length values than the random distribution. As the uniform distributions are less obstructive to diffusion, tracers reach earlier to the final normal diffusion regime. It should be noted that when the obstacle separation is 1 the crossover length tends to a value that is the equatorial distance from a site situated in front the obstacle and the site situated behind, that is, the obstacle diameter plus 1 . It should be noticed that the $r^{*}$ values for mobile obstacles are greater (tend to the obstacle diameter plus 2) than in the uniform obstacle distributions. The reason is that in the second case all the obstacles (immobile) are separated from the others, whereas in the first case clusters of obstacles may be formed yielding a bigger average obstacle size.

\section{Conclusions}

The ability to simulate diffusion in complex media is an essential first step for understanding small particle dynamics in living cells. Although there is a lot of information published in literature about diffusion in crowded $2 \mathrm{D}$ media, there is a 
shortage of systematic studies concerning three-dimensional crowding. 3D studies are particularly interesting because they allow to asses the real importance of the crowding in diffusion without the influence of the low-dimensional restrictions.

The diffusion process in these media is well described by the $\log \left(<r^{2}>/ t\right)$ versus $\log (t)$ curves. They show that the diffusion coefficient changes with time during a period of anomalous diffusion until it reaches a long-time constant value that corresponds to a normal diffusion in a denser homogeneous medium. Our simulations reveal how these curves are affected by the density, size, mobility and spatial distribution of obstacles. Parameters such as the anomalous diffusion exponent (that indicates the degree of this time dependence), the diffusion coefficient for long-time normal diffusion, and the crossover time from the anomalous diffusion regime to normal one are used to characterize the crowding effect on diffusion.

Our simulation results indicate that increasing the obstacle site density increases the importance of the anomalous diffusion by increasing the reduction rate of the diffusion coefficient with time (smaller $\alpha$ value) and reducing the diffusion coefficient $\left(D^{*}\right)$ corresponding the long-time normal diffusion. We have also found that the dependence of the crossover time on the obstacle density shows a different behaviour depending on the size of the obstacles (that is, the particle-obstacle relative size). In contrast to what happens with small size obstacles and in accordance with what it is observed in $2 \mathrm{D}$ simulations ${ }^{40}$, for big obstacles the crossover time decreases with the obstacle density. These differences are a consequence of the different spatial pattern distribution of the excluded volume shown for each obstacle size.

A new contribution of the present work is the analysis of the spatial distribution pattern of the excluded volume due to obstacles and how this pattern affects the particle diffusion. Our results show that for a fixed density of sites occupied by obstacles, the effect on diffusion is different depending on how this 
obstacle-excluded volume is partitioned. When a same total obstacle site density is distributed in a great number of small size obstacles the anomalous diffusion behaviour is more enhanced (faster decrease of the diffusion coefficient, smaller crossover time and smaller long-time diffusion coefficient) than when this excluded volume is partitioned into a small number of big size obstacles. This effect can be understood taking into account that the first case implies a great number of local volumes with empty sites separated by a great number of obstacles that makes more difficult the free displacements of the diffusing particles. On the contrary, in a pattern with big size obstacles the free space is divided into a smaller number of big size empty zones separated by a small number of diffusional barriers. Our results confirm that the spatial distribution pattern of the excluded volume plays an important role in the anomalous diffusion processes. In the estimation of the diffusion coefficient in crowded media it is not enough to take into account only the total excluded volume, its spatial distribution pattern must also be considered.

Our results confirm that when the obstacles are mobile its obstructive effect is diminished. We found that in increasing the value of the mobility factor, the anomalous exponent increases and the crossover time diminishes. In consequence, the importance of the anomalous diffusion regime is reduced.

Another important observation is that a uniform distribution of obstacles is less obstructive to diffusion in comparison to a random distribution of immobile obstacles being its results similar to those of mobile obstacle distributions. The structural organization of the system presenting elements such as "channels" gives to the diffusing particles a continuum path of empty sites to move. With the uniform distributions the rate reduction of the diffusion coefficient is smaller, the crossover time to normal diffusion appears earlier and the limiting diffusion coefficient is higher 
than with a random distribution. An example of such a system is the cell cytoplasm which contains microtubules were small particles diffuse.

\section{Acknowledgements}

We acknowledge the financial support from: CNCSIS 551 Romania, Spanish Ministry of Science and Innovation (Projects UNBA05-33-001 and CTM2006-13583) and "Comissionat d'Universitats i Recerca de la Generalitat de Catalunya" (2005SGR00616, 2009SGR-00465 and XRQTC). IP thanks the Juan de la Cierva Program of the Spanish Ministry of Science. 


\section{Notes and references}

1. A. B. Fuklton, Cell, 1982, 30, 345-347.

2. S. B. Zimmerman and A. P. Minton, Annu. Rev. Biophys. Biomol. Struct., 1993, 22, 27-65.

3. A. P. Minton, J. Biol. Chem., 2001, 276, 10577-10580.

4. R. Kopelman, Science, 1988, 241, 1620-1626.

5. D. Ben-Avraham and S. Havlin, in Diffusion and reactions in fractals and disordered systems. Cambridge University Press. Cambridge (2000)

6. R. J. Ellis, Trends. Biochem. Sci., 2001, 26, 597-604.

7. R. J. Ellis and A. P. Minton, Nature, 2003, 425, 27-28.

8. S. Schnell and T. E. Turner, Prog. Biophys. Mol. Biol., 2004, 85, 235-260.

9. B. K. Derham and J. J. Harding, $B B A$, 2006, 1764, 1000-1006.

10. R. Grima and S. Schnell, Biophys. Chem., 2006, 124, 1-10.

11. M. Agrawal, S. B. Santra, R. Anand and R. Swaminathan, PRAMANA J. Phys., 2008, 71, 359-368.

12. H. X. Zhou, G. Rivas and A. P. Minton, Annu. Rev. Biophys., 2008, 37, 375-395.

13. J. A. Dix and A. S. Verkman, Annu. Rev. Biophys., 2008, 37, 247-263.

14. M. J. Saxton, Biophys. J., 2008, 94, 760-771.

15. H. X. Zhou, J. Phys. Chem. B., 2009, 113, 7995-8005.

16. T. J. Feder, I. Brust-Mascher, J. P. Slattery, B. Baird and W. W. Webb, Biophys. $J .$, 1996, 70, 2767-2773.

17. O. Seksek, J. Biwersi and A. S. Verkman, J. Cell Biol., 1997, 138, 131-142.

18. N. Periasamy and A. S. Verkman, Biophys. J., 1998, 75, 557-567.

19. M. Ario-Dupont, G. Foucault, M. Vacher, F. Devaux and S. Cribier, Biophys. J., 2000, 78, 901-907. 
20. M. Platani, I. Goldberg, J. R. Swedlow and A. I. Lamond, J. Cell Biol., 2000, 151, 1561-1574.

21. E. O. Potma, W. P. de Boeij, L. Bosgraaf, J. Roelofs, P. J. M. Van Haastert and D. A. Wiersma, Biophys. J., 2001, 81, 2010- 2019.

22. A. S. Verkman, Science, 2002, 27, 27-33.

23. Y. Cheng, R. K. Prud'homme and J. L. Thomas, Macromolecules, 2002, 35, 8111- 8121.

24. M. Platani, I. Goldberg, A. I. Lamond and J. R. Swedlow, Nat. Cell Biol., 2002, 4, $502-508$

25. M. Wachsmuth, T. Weidemann, G. Muller, M. W. Hoffman-Rohrer, T. A. Knoch, W. Waldeck and J. Langowski, Biophys. J., 2003, 84, 3353-3363.

26. N. Fatin-Rouge, K. Starchev and J. Buffle, Biophys. J., 2004, 86, 2710-2719.

27. E. Dauty and A. S. Verkman, J. Molecular Recognition, 2004, 17, 441-447.

28. M. Weiss, M. Elsner, F. Kartberg and T. Nilsson, Biophys. J., 2004, 87, 35183824.

29. K. Murase, T. Fujiwara, Y. Umemura, K. Suzuki, R. Iino, et al., Biophys. J, 2004, 86, 4075-4093.

30. A. Kusumi, H. Ike, C. Nakada, K. Murase and T. Fujiwara, Semin. Immunol., $2005,17,3-21$

31. D. S. Banks and C. Fradin, Biophys. J., 2005, 89, 2960-2971.

32. A. Masuda, K. Ushida and T. Okamoto, Biophys. J., 2005, 88, 3584-3591.

33. H. Sanabria, Y. Kubota and M. N. Waxhan, Biophys. J., 2007, 92, 313-322.

34. M. Guigas, C. Kalla and M. Weiss, Biophys. J., 2007, 93, 316-323.

35. I. Pastor, E. Vilaseca, S. Madurga, J. L. Garcés, M. Cascante and F. Mas, J. Phys. Chem. B, 2010, 114, 4028-4034.

36. M. J. Saxton, Biophys. J., 1987, 52, 989-997. 
37. M. J. Saxton, Biophys. J., 1990, 58, 1303-1306.

38. H. P. Kao, J. R. Abney and A. S. Verkman, J. Cell Biol., 1993, 120, 175-184.

39. M. J. Saxton, Biophys. J., 1993, 64, 1053-1062.

40. M. J. Saxton, Biophys. J., 1994, 66, 394-401.

41. M. J. Saxton, Biophys. J., 1996, 70, 1250-1262.

42. P. A. Netz and T. Dorfmuller, J. Chem. Phys., 1995, 103, 9074-9082.

43. B. P. Olveczki and A. S. Verkman, Biophys. J., 1998, 74, 2722-2730.

44. A. Gil, J. Segura, J. A. G. Pertusa and B. Soria, Biophys. J., 2000, 78, 13-33.

45. Y. Tang, T. Schlumpberger, T. Kim, M. Lueker and R. S. Zucker, Biophys. J., 2000, 78, 2735-2751.

46. K. Lipkow, S. S. Andrews and D. Bray, J. of Bacteriology, 2005, 187, 45-53.

47. J. A. Dix, E. F. Hom and A. S. Verkman, J. Phys. Chem. B, 2006, 110, 18961906.

48. C. Echeveria, K. Tucci and R. Kapral, J. Phys. Condens. Matter., 2007, 19, 065146-065158.

49. A. Isvoran, E. Vilaseca, J. L. Garces, L. Unipan and F. Mas, Proceedings of the 6th Conference of Balkan Physics Union, A.I.P., 2007, 889, 469-470.

50. A. Isvoran, E. Vilaseca, L. Unipan, J. L. Garces and F. Mas, Rom. Biophys. J., $2007,17,21-3$

51. M. J. Saxton, Biophys. J., 2007, 92, 1178-1191.

52. A. Isvoran, E. Vilaseca, L. Unipan, J. L. Garces and F. Mas, Rev. Rom. Chem., 2008, 53, 415-419.

53. G. Guigas and M. Weiss, Biophys. J., 2008, 94, 90-94.

54. S. Havlin and D. Ben-Avraham, Adv. Phys., 1987, 36, 695-798.

55. J. P. Bouchaud and A. Georges, Phys. Rep., 1990, 185, 127-293.

56. R. Metzler and J. Klafter, Phys. Rep., 2000, 339, 1-77. 
Table 1 Lattice sizes, obstacle sizes and obstacle excluded volume $(\phi)$ considered in the simulations of tracer diffusion in lattices with uniformly distributed obstacles. For each uniform distribution the separation between two nearest-neighbour obstacles is also given.

\begin{tabular}{|c|c|c|c|}
\hline Obstacle size & $\phi$ & Obstacle separation & Lattice size \\
\hline \multirow{2}{*}{1 site $(1 \times 1 \times 1)$} & 0.037 & 2 sites & $99 \times 99 \times 99$ \\
\cline { 2 - 4 } & 0.125 & 1 site & $100 \times 100 \times 100$ \\
\hline \multirow{3}{*}{27 sites $(3 \times 3 \times 3)$} & 0.125 & 3 sites & $102 \times 102 \times 102$ \\
\cline { 2 - 4 } & 0.216 & 2 sites & $100 \times 100 \times 100$ \\
\cline { 2 - 4 } & 0.422 & 1 site & $100 \times 100 \times 100$ \\
\hline \multirow{2}{*}{81 sites $(5 \times 5 \times 5 \mathrm{R})$} & 0.236 & 2 sites & $105 \times 105 \times 105$ \\
\cline { 2 - 4 } & 0.375 & 1 site & $102 \times 102 \times 102$ \\
\hline \multirow{2}{*}{$179 \operatorname{sites}(7 \times 7 \times 7 \mathrm{R})$} & 0.246 & 2 sites & $99 \times 99 \times 99$ \\
\cline { 2 - 4 } & 0.350 & 1 site & $104 \times 104 \times 104$ \\
\hline
\end{tabular}


Table 2 Upper limit estimation of the initial diffusion coefficient values, $D_{0}{ }^{\prime}(\phi)$, obtained in all the simulations performed with immobile random distributed obstacles. The value of the log-time diffusion coefficient, $D^{*}$, is also shown.

\begin{tabular}{|c|c|c|c|c|}
\hline Obstacle size & $\phi$ & $1-$ collision probability & $D_{0}{ }^{\prime}(\phi)$ & $D^{*}(\phi)$ \\
\hline \multirow{4}{*}{$1 \times 1 \times 1$} & 0.1 & 0.900 & 0.878 & 0.834 \\
\hline & 0.2 & 0.800 & 0.768 & 0.679 \\
\hline & 0.3 & 0.700 & 0.661 & 0.521 \\
\hline & 0.4 & 0.600 & 0.557 & 0.362 \\
\hline \multirow{4}{*}{$3 \times 3 \times 3$} & 0.1 & 0.967 & 0.948 & 0.898 \\
\hline & 0.2 & 0.934 & 0.901 & 0.808 \\
\hline & 0.3 & 0.901 & 0.847 & 0.706 \\
\hline & 0.4 & 0.867 & 0.787 & 0.605 \\
\hline \multirow{4}{*}{$5 \times 5 \times 5 R$} & 0.1 & 0.969 & 0.956 & 0.906 \\
\hline & 0.2 & 0.938 & 0.916 & 0.822 \\
\hline & 0.3 & 0.907 & 0.867 & 0.732 \\
\hline & 0.4 & 0.876 & 0.809 & 0.633 \\
\hline \multirow{4}{*}{$7 \times 7 \times 7 R$} & 0.1 & 0.973 & 0.962 & 0.913 \\
\hline & 0.2 & 0.945 & 0.929 & 0.833 \\
\hline & 0.3 & 0.918 & 0.889 & 0.749 \\
\hline & 0.4 & 0.890 & 0.840 & 0.659 \\
\hline
\end{tabular}




\section{Captions for figures}

Fig. 1 The four obstacle sizes considered in the MC simulations: a) 1 site, b) 27 sites $(3 \times 3 \times 3)$, c) 81 sites $(5 \times 5 \times 5 R)$ and d) 179 sites $(7 \times 7 \times 7 R)$.

Fig. $2 \log \left(<r^{2}>/ t\right)$ versus $\log (t)$ curves for diffusion in 3D crowded media with 81-site obstacles $(5 \times 5 \times 5 \mathrm{R})$ presenting two different site density occupancies $(\phi=0.3,0.4)$. The curve for the unobstructed medium is also added. The regions corresponding to the different diffusion behaviours and the manner to determine the characteristic parameters are illustrated.

Fig. 3 Plot of $\left\langle r^{2}>\right.$ vs. time in 4 systems with immobile obstacles randomly distributed having a same obstacle excluded volume (0.4) but different obstacle sizes: 1 site $(1 \times 1 \times 1), 27$ sites $(3 \times 3 \times 3), 81$ sites $(5 \times 5 \times 5 R)$ and 179 sites $(7 \times 7 \times 7 R)$. The arrow indicates the crossover time position.

Fig. 4 Dependence of a) the anomalous diffusion exponent, $\alpha, b)$ the long-time diffusion coefficient, $\left.D^{*}, \mathrm{c}\right)$ the crossover time, $\tau$, and d) the crossover length, $\mathrm{r}^{*}$, on the obstacle excluded volume for four different obstacle sizes: 1-site obstacles

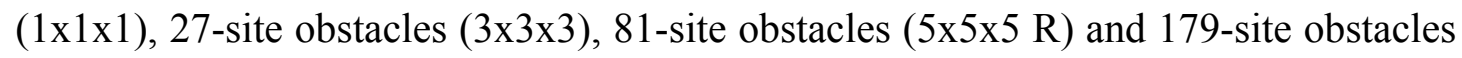
$(7 \times 7 \times 7 \mathrm{R})$. Error estimations for $\alpha$ and $D^{*}$ values are smaller than \pm 0.001 and \pm 0.00002 , respectively. For the crossover time and the crossover length the error interval is shown by the vertical bars.

Fig. 5 Plots showing the obstacle density distribution around an average empty site. For each for obstacle size the curves corresponding to the four excluded volumes $(0.1$, $0.2,0.3$ and 0.4 ) are plotted. In every curve the position of the crossover length ( $\left.\mathrm{r}^{*}\right)$ is marked with a circle. 
Fig. $6 \log \left(<r^{2}>/ t\right)$ versus $\log (t)$ curves for diffusion in a 3D crowded system with an obstacle excluded volume of 0.3 , obstacles having a size of 81 sites $(5 \times 5 \times 5 R)$ and presenting five different mobilities according to the probability factor M.

Fig. 7 Dependence of a) the anomalous diffusion exponent, $\alpha, b)$ the long-time diffusion coefficient, $\left.D^{*}, \mathrm{c}\right)$ the crossover time, $\tau$, and d) the crossover length, $r^{*}$, on the obstacle excluded volume for four different size obstacles having different mobility: 1-site obstacles ( $M=1)$, 27-site obstacles $(M=0.75)$, 81-site obstacles $(\mathrm{M}=0.25)$ and 179 -site obstacles $(\mathrm{M}=0)$. Error estimations for $\alpha$ and $D^{*}$ values are smaller than \pm 0.001 and \pm 0.00002 , respectively. For the crossover time and the crossover length the error interval is shown by the vertical bars.

Fig. $8 \log \left(<r^{2}>/ t\right)$ versus $\log (t)$ curves for diffusion in lattices with immobile obstacles having 81 sites size $(5 \times 5 \times 5 \mathrm{R})$. For $\phi=0.236$ the curves corresponding to the random distribution and an uniform distribution with 2 empty sites between nearestneighbours are shown. For $\phi=0.375$ the curves correspond to the random distribution and a uniform distribution with 1 empty site between nearest-neighbours.

Fig. 9 Dependence of a) the anomalous diffusion exponent, $\alpha, b)$ the long-time diffusion coefficient, $\left.D^{*}, \mathrm{c}\right)$ the crossover time, $t$, and d) the crossover length, $\mathrm{r}^{*}$, on the obstacle excluded volume for four different size obstacles: $1 \times 1 \times 1$ (circles), $3 \times 3 \times 3$ (squares), 5x5x5 R (up triangles) and $7 \times 7 \times 7 \mathrm{R}$ (down triangles). For each obstacle size two spatial distributions are considered: uniform (continuous line) and random (dotted line). The obstacles in the random distributions are immobile. For the uniform distributions the values of the nearest-neighbours separation (sep) are indicated in a) and $b)$. 
Figure 1

a)

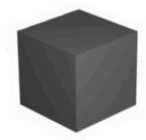

c)

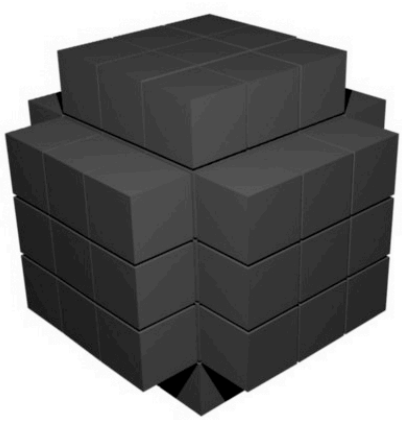

b)

d)

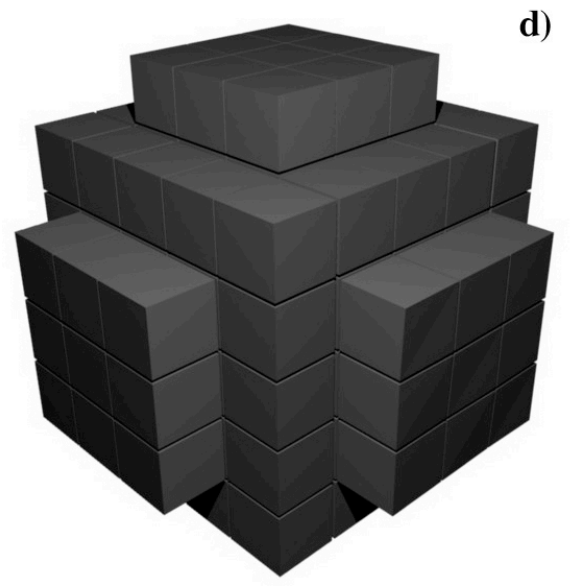


Figure 2 
Figure 3

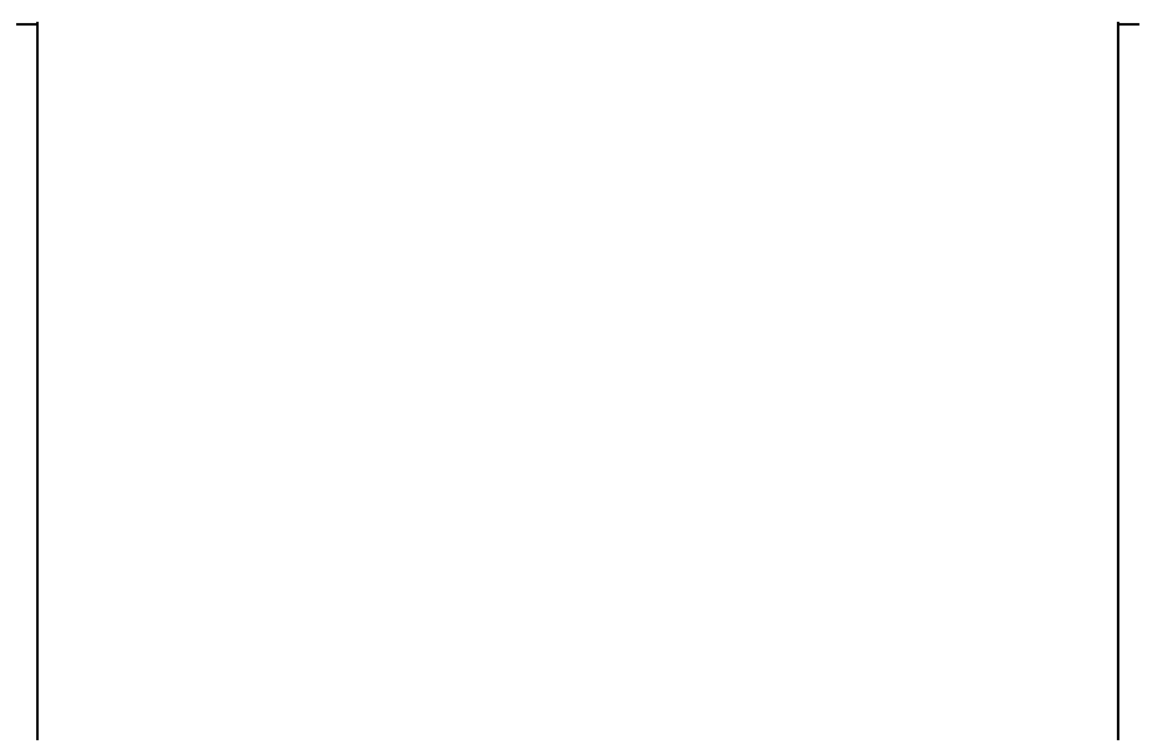


Figures $4 a$ and $4 b$ 
Figures $4 c$ and $4 d$
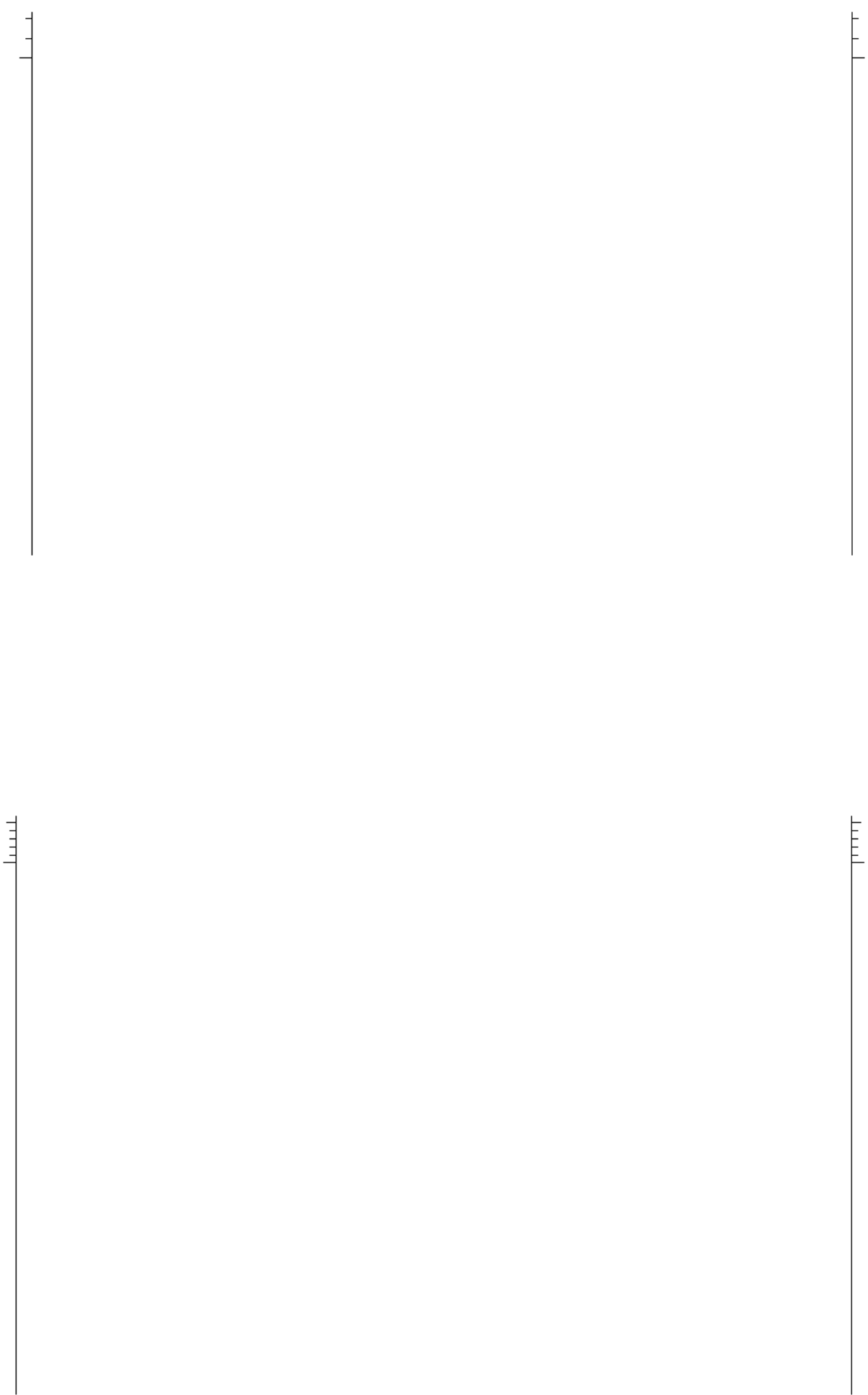
Figures 5a and 5b

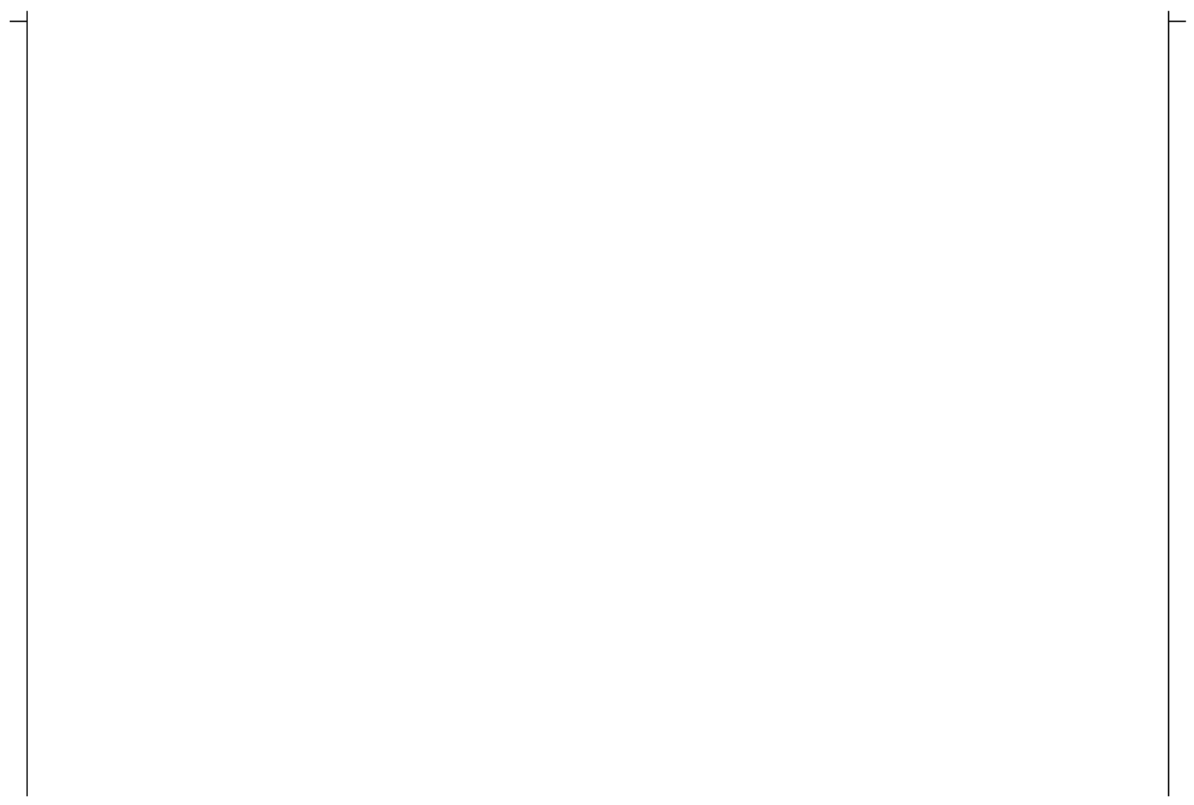


Figures 5c and 5d

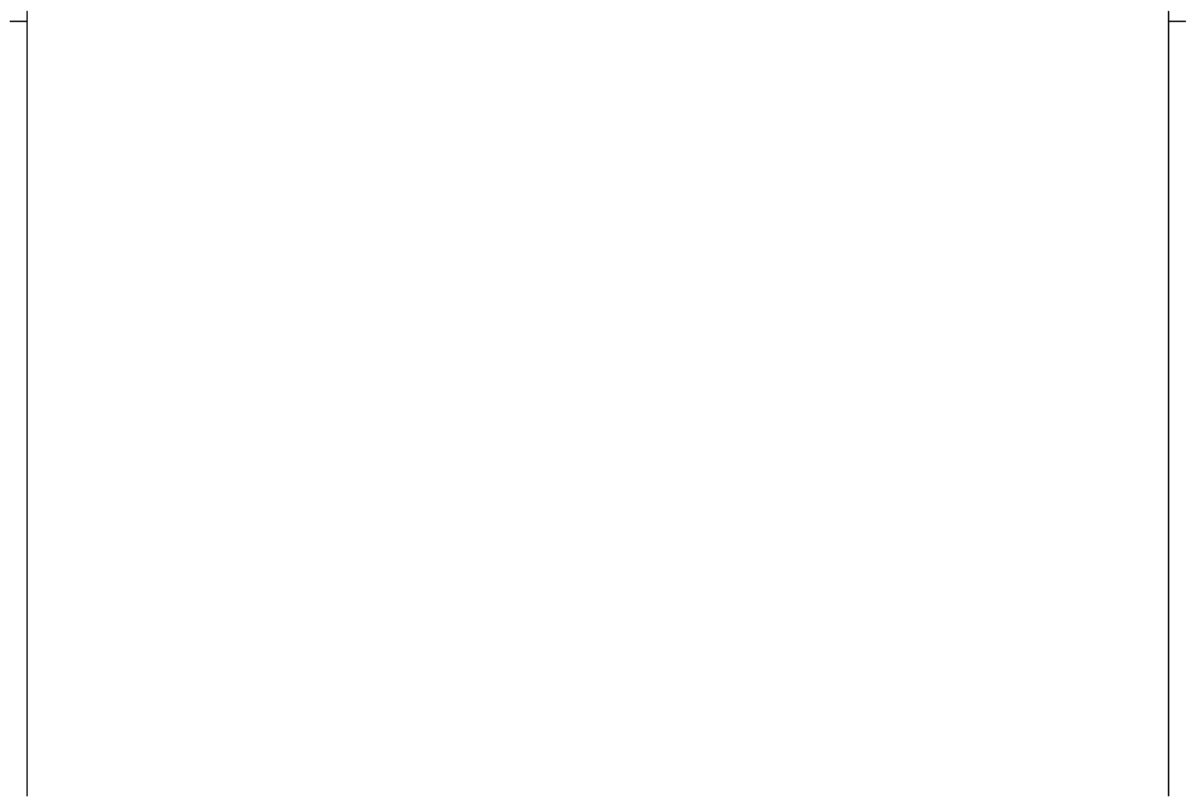


Figure 6

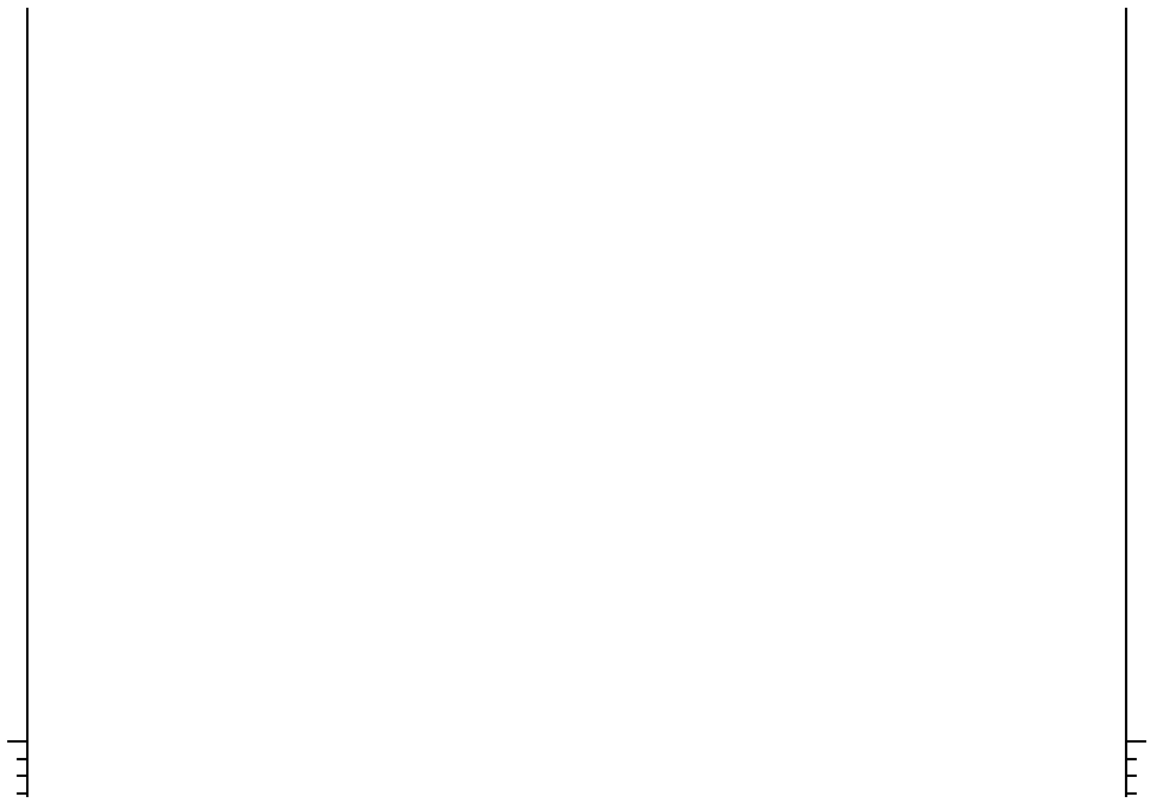


Figures $7 a$ and $7 b$
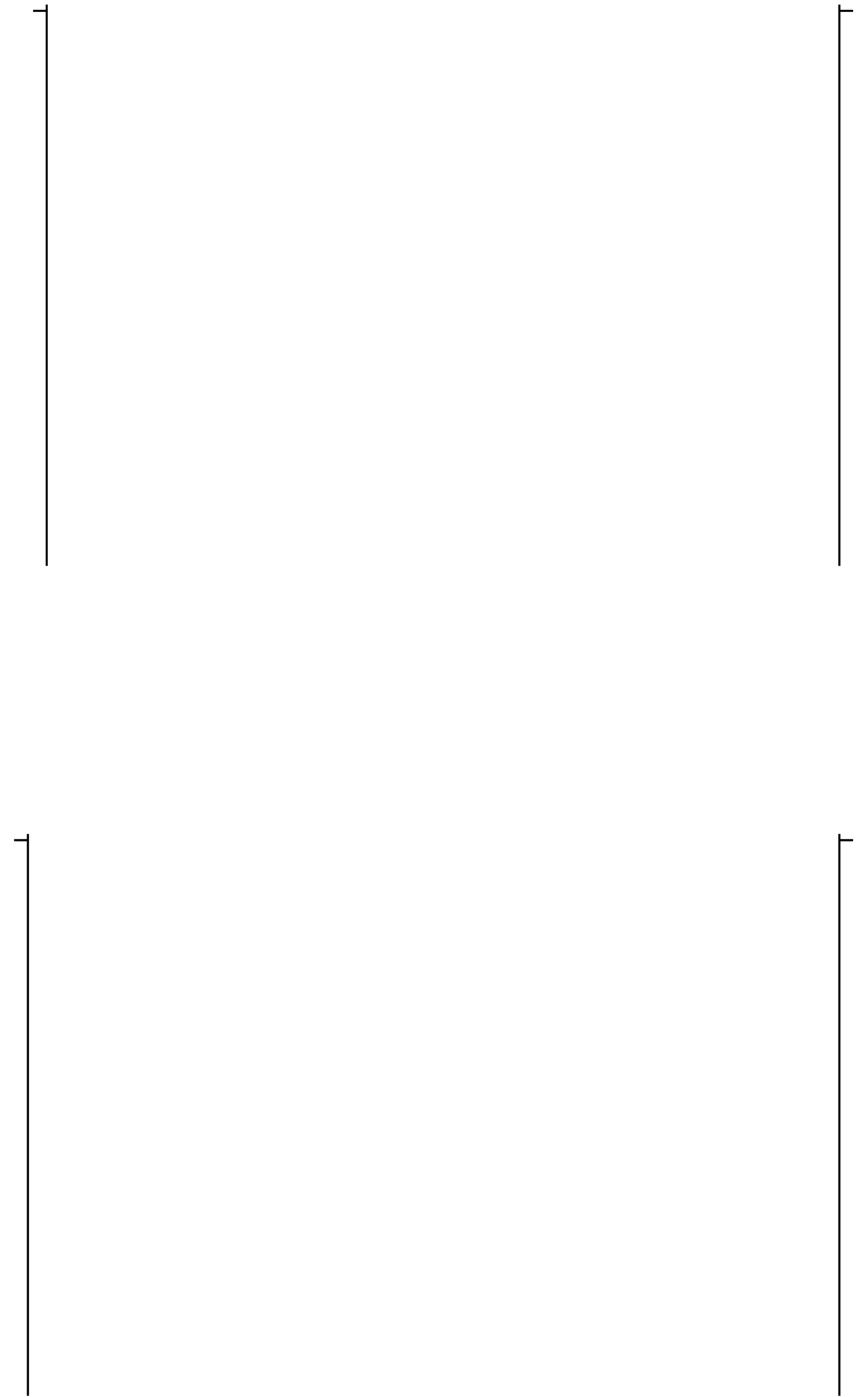
Figures $7 c$ and $7 d$
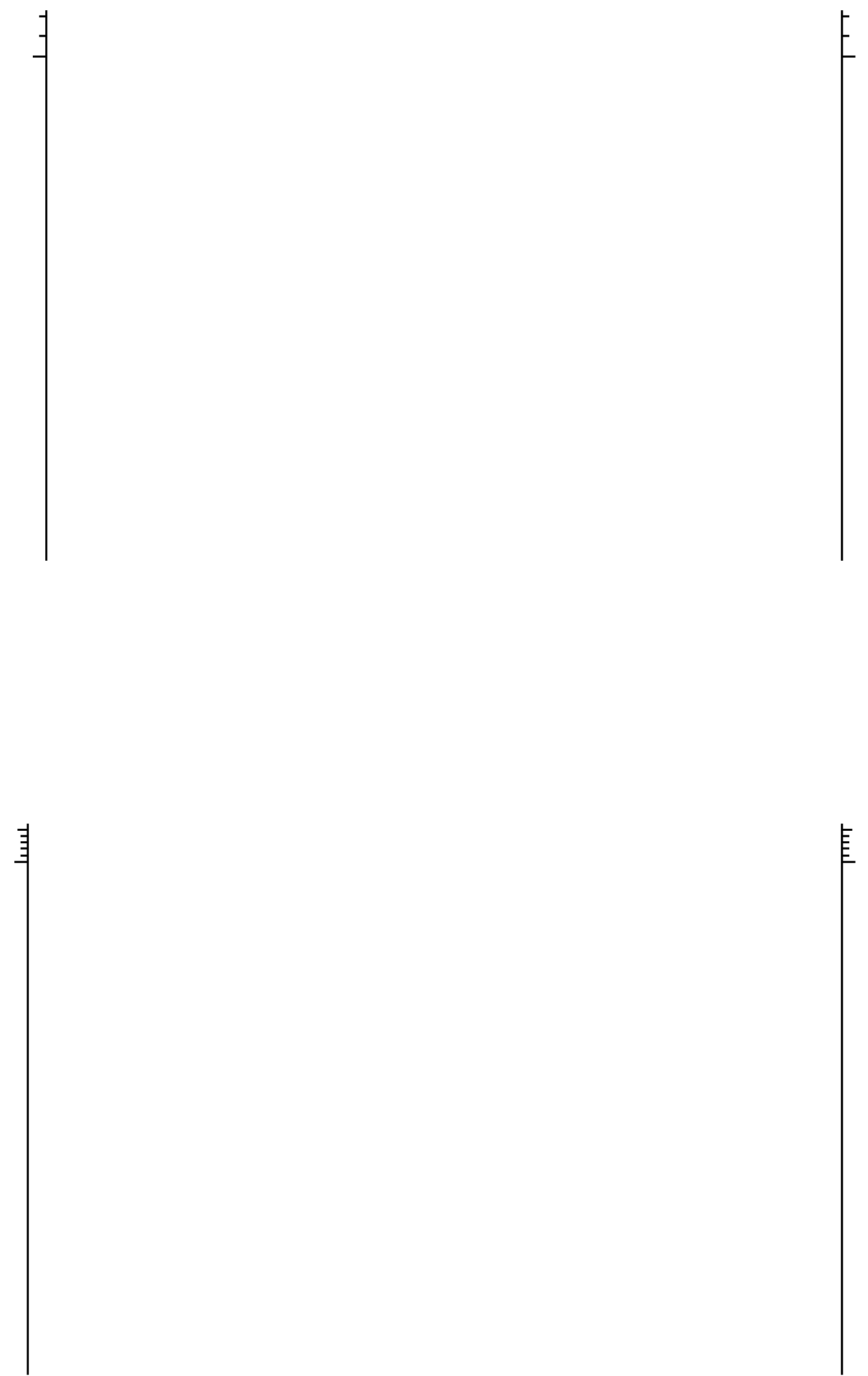
Figure 8

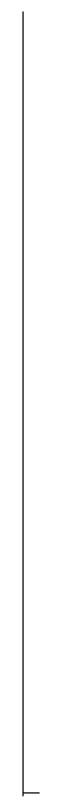


Figures 9a and 9b
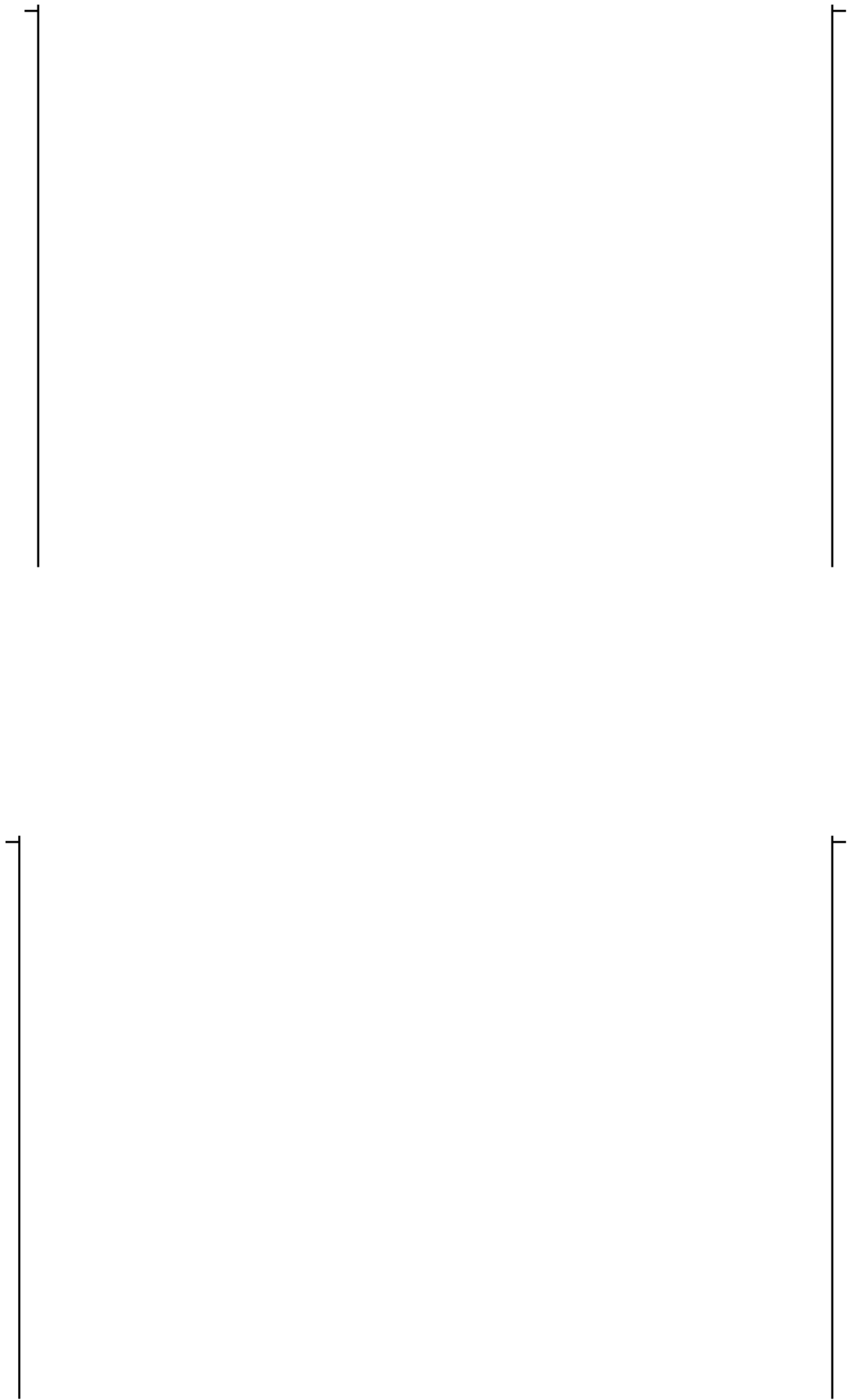
Figures 9c and 9d
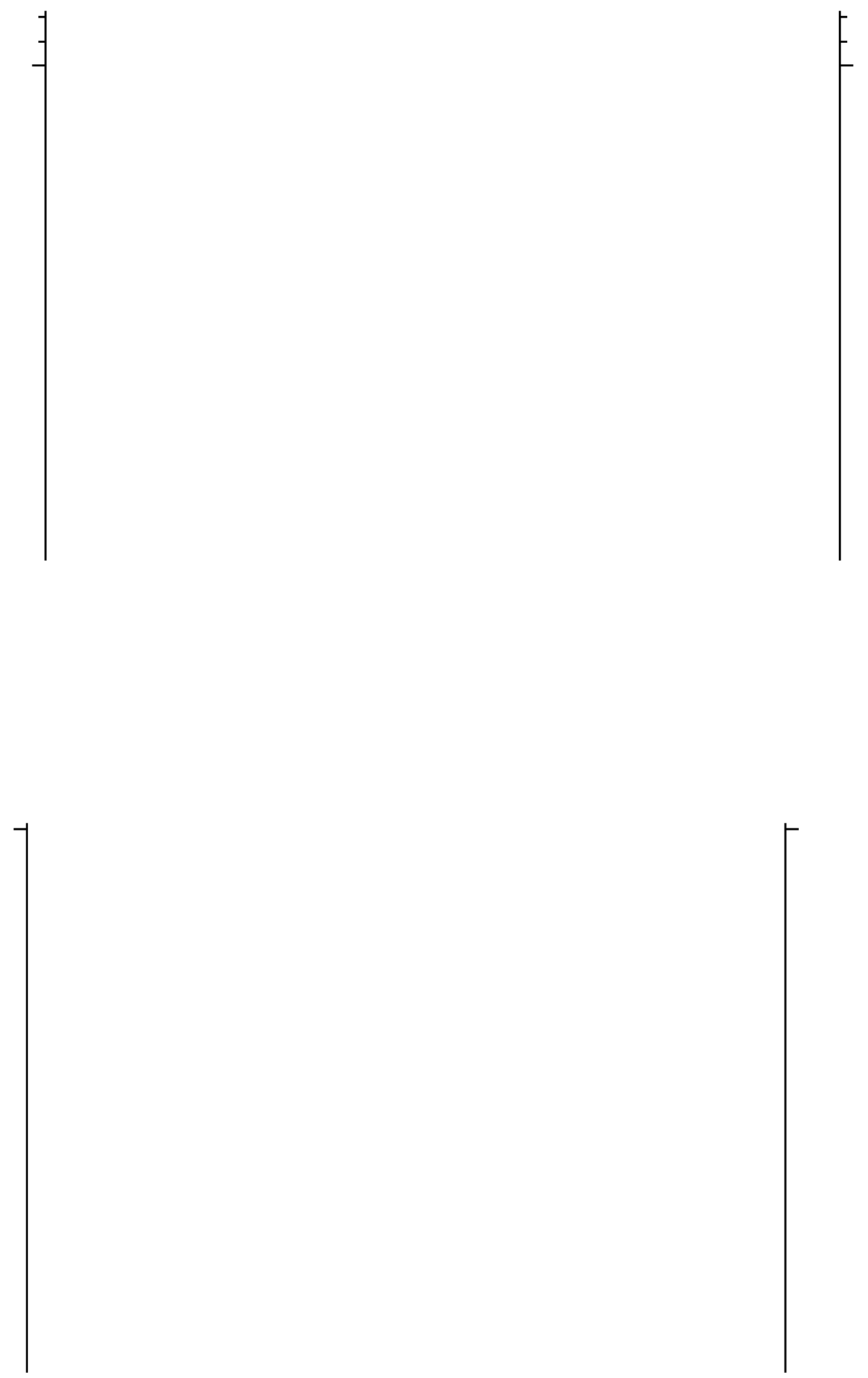